\title{
The prognostic impact of neutrophil to lymphocyte ratio in advanced non-small cell lung cancer patients treated with EGFR TKI
}

This article was published in the following Dove Press journal: International Journal of General Medicine

\author{
Thang Thanh Phan' \\ Toan Trong Ho' \\ Hue Thi Nguyen' \\ Hang Thuy Nguyen ${ }^{2}$ \\ Thu Bich Tran ${ }^{3}$ \\ Son Truong Nguyen' \\ 'Laboratory D Unit, Clinical Cancer \\ Center, Cho Ray Hospital, Ho Chi \\ Minh City, Vietnam; ${ }^{2}$ Pathology \\ Department, Cho Ray Hospital, Ho \\ Chi Minh City, Vietnam; ${ }^{3}$ Faculty of \\ Biology and Biotechnology, University \\ of Science, VNU-HCM, Ho Chi Minh \\ City, Vietnam
}

Purpose: To identify and clarify the roles of inflammatory markers in prognosis for advanced non-small cell lung cancer (NSCLC) patients treated with EGFR tyrosine kinase inhibitor (TKI). Patients and methods: One hundred and twelve adenocarcinoma, clinical stage IV, NSCLC patients with either EGFR exon 19 deletion (E19del) or EGFR exon 21 L858R substitution mutation (L858R) were selected for this study. The blood cell count at different stages of treatment was used to calculate the inflammatory markers. The Kaplan-Meier statistics and Cox regression model were used to test the differences of progression-free survival (PFS) between groups by the optimal cutoff point of biomarkers.

Results: The median values of white blood cell (WBC), neutrophil to lymphocyte ratio (NLR), monocyte to lymphocyte ratio (MLR) and platelet to lymphocyte ratio (PLR) in NSCLC patients tended to be reduced after 3 months treated with EGFR TKI and increased conversely when the disease develops progression $(P<0.001)$. With an optimal cutoff point of 2.96 , NLR is the best prognostic marker in prediction of clinical response among the investigated markers (area under the curve $[\mathrm{AUC}]=0.873,95 \% \mathrm{CI}: 0.821-0.926, P<0.001)$, and it is an independent predictive marker $(\mathrm{OR}=3.52,95 \% \mathrm{CI}: 1.42-8.71, P<0.001)$. With optimal cutoff point of 0.38 , MLR is also a predictive marker in response evaluation (AUC $=0.762,95 \%$ CI: $0.691-0.832$ ). Univariate analyses have shown that the larger tumor size $(>3 \mathrm{~cm})$ and the high level of pretreatment NLR were associated with the shortening of PFS (HR=2.24, 95\% CI: $1.04-4.83, P=0.039$ and $\mathrm{HR}=2.67,95 \% \mathrm{CI}: 1.41-5.03, P=0.006$, respectively). Multivariate analysis has shown that the elevated NLR is an independent prognostic marker for worse PFS of NSCLC patients treated with EGFR TKI (HR=2.15, 95\% CI: $1.15-3.99, P=0.016)$.

Conclusion: NLR and MLR are valuable markers in response evaluation for NSCLC patients treated with EGFR TKI. The elevated NLR is also an independent prognostic factor for worse survival.

Keywords: neutrophil to lymphocyte ratio, NLR, NSCLC, EGFR TKI

\section{Introduction}

Lung cancer is a leading cause of death from cancer worldwide, mostly non-small cell lung cancer (NSCLC). ${ }^{1}$ This cancer develops silently and has no specific symptoms. Almost all patients were diagnosed in advanced stages (IIIB and IV). Treatment by EGFR tyrosine kinase inhibitor (TKI) helps to enhance the response rate, prolong the progression-free survival (PFS) and overall survival (OS) for NSCLC patients. ${ }^{2}$ In order to monitor the treatment for patients, several biomarkers in serum such as cyfra 21-1 (cytokeratin 19), CA12-5 (cancer antigen 125) or NSE (neuron specific enolase) are widely used but with limited sensitivity and specificity. ${ }^{3}$ However, computerized tomography-scanner
Correspondence: Son Truong Nguyen Department of General Director, Cho Ray Hospital, 20IB Nguyen Chi Thanh Street, Dist. 5, Ho Chi Minh City 700000, Vietnam

Tel +842838554137

Fax +84283855 7267

Email truongson_cr@yahoo.com.vn 
(CT), positron emission tomography-computed tomography and MRI are also frequently used diagnostic tests, but they are expensive and carry potential risk from radioactive rays.

Many studies have shown the roles of systematic inflammation markers which help to monitor the response to chemotherapy, radiotherapy or surgery and the prognosis for NSCLC patients. ${ }^{4-12}$ The number of granulocytes and platelets (PLTs) are increased in the peripheral blood of patients that make an increase of neutrophil to lymphocyte ratio (NLR), monocyte to lymphocyte ratio (MLR) or platelet to lymphocyte ratio (PLR). These ratios are indirect markers, which indicate tumor cell activity, and can be considered the basis of above evaluations. ${ }^{4-12}$

A few studies mention the role of these markers on targeted treatment and prognosis for NSCLC patients but with contradictory results. ${ }^{13-18}$ The results of Lin et $a l,{ }^{13}$ Ding et a ${ }^{14}$ and Meriggi et $\mathrm{al}^{15}$ have shown that the high level of pretreatment NLR was seen to be an independent prognostic factor for poor outcome (both PFS and OS) in EGFR-mutated NSCLC patients treated with first-line EGFR TKI. Conversely, the results of Minami et al's study have shown that the elevated NLR is an independent prognostic factor for OS, but not for PFS in NSCLC. ${ }^{16}$ Other studies with two groups correspond to two treatment methods, Sim et al concluded that the pretreatment NLR was a significant prognostic factor for the chemotherapy group but not for the EGFR TKI therapy group. ${ }^{18}$ In prognosis of PFS for NSCLC patients treated with EGFR TKI using lymphocyte to monocyte ratio (LMR) marker, Chen et a ${ }^{17} \mathrm{dem}$ onstrated that the high LMR is an independent predictive factor for a longer PFS, which is completely different to the results of the Minami et $\mathrm{al}^{16}$ study. On that basis, our study aimed to identify and clarify the roles of inflammatory markers such as white blood cell (WBC), PLT, NLR, MLR and PLR in predicting response to EGFR TKI and prognosis for NSCLC patients.

\section{Patients and methods}

\section{Patients and parameters}

The study was conducted on 112 NSCLC patients with either EGFR E19del or L858R mutation who were treated with EGFR TKI (standard dose of erlotinib or gefitinib) from December 2015 to March 2018 at Cho Ray hospital. The NSCLC patients were under a treatment evaluation based on the RECIST v1.1 criteria. ${ }^{19}$ The number of WBC, neutrophil (NEU), lymphocyte (LYM), monocyte (MONO) and PLT of NSCLC patients at different stages, before treatment, after 3 months of treatment with EGFR TKI, and at progressive disease (PD) stage were recalled from the laboratory database and the markers of NLR, MLR and PLR calculated. The blood cell analysis was performed on UniCel DxH 800 (Beckman Coulter, CA, USA). The marker values of 112 healthy adults (with age and gender similar to the patients) were also used for comparison with the marker values of NSCLC patients posttreatment to assess the reduction level of inflammatory markers in NSCLC patients after a course of treatment. Healthy disease-free adults, who have annual medical examination (AME), were chosen who have the class-I health certificate according to the AME criteria of Cho Ray hospital. The other parameters were retrieved retrospectively from medical records. This study was considered and approved by the Ethics Committees of Cho Ray hospital (approval number: $602 / \mathrm{CN}-\mathrm{HDDD}$ ). The authors were permitted to access the clinical records and laboratory database to select data for the study with the commitment of information confidentiality, therefore, patient consent was not required.

\section{Statistical analysis}

The Fisher's exact test was used to compare the relative frequencies, whereas the Kruskal-Wallis rank test was used to compare the median value of each marker between groups. The logistic regression model was used to construct the receiver operating characteristic (ROC) curve and define the optimal cutoff point of each marker, together with sensitivity, specificity, and the value under the ROC curve (area under the curve $[\mathrm{AUC}]$ ) in predicting the response to therapy. The median PFS based on subgroups of the inflammatory markers and clinical characteristics were estimated by the KaplanMeier statistics with $95 \%$ CI. The association of clinical outcome with levels of markers and clinical characteristics were tested by the Cox regression model and calculated the HR with $95 \%$ CI. All data analyses were performed on STATA 14.0 statistical software (StataCorp LP, College Station, TX, USA). $P<0.05$ was considered statistically significant.

\section{Ethics statement}

This study was considered and approved by the ethics committees of Cho Ray Hospital (approval number: 602/CNHDDD). Patient consent was not required.

\section{Results}

\section{Patient characteristics}

A total of 112 adenocarcinoma, clinical stage IV, NSCLC patients with either EGFR E19del (77 cases) or L858R (35 cases) mutation, and 112 healthy adults with similar age and gender were selected for this study. Patients were categorized into four groups based on the level (below vs above cutoff value) of NLR (2.96) and MLR (0.38) at baseline: 29 patients 
with NLR $<2.96$ and MLR $<0.38 ; 7$ patients with $\mathrm{NLR}<2.96$ and MLR $\geq 0.38 ; 22$ patients with NLR $\geq 2.96$ and MLR $<0.38$ and 54 patients with NLR $\geq 2.96$ and MLR $\geq 0.38$. The characteristics of patients were presented in Table 1 .

The median age of all patients was 59 (from 33 to 88 years old). Of 112 patients, 61 (54.5\%) were female, and 51 male (45.5\%). In overall assessment, 17 patients (15.2\%) with severe symptoms were scored with the Eastern Cooperative Oncology Group performance status (ECOG PS) $\geq 2$ who are unable to carry out any work. Most of the patients have $\geq 2$ distant metastasis sites $(89 / 112,79.5 \%)$, and tumor size $>3 \mathrm{~cm}$ (87/112, 77.7\%). The groups with NLR $\geq 2.96$ have the higher absolute count of NEU $(P<0.001)$ and MONO $(P=0.025)$, but have the lower absolute count of LYM $(P<0.001)$ compared with the values of groups with NLR $<2.96$ (Table 1).

Eighty-nine cases (79.5\%) were treated with EGFR TKI as first-line treatment, whereas 23 cases $(20.5 \%)$ were treated with EGFR TKI after surgery with or without radiotherapy,

Table I Patient characteristics

\begin{tabular}{|c|c|c|c|c|c|c|}
\hline \multirow[t]{2}{*}{ Characteristics } & \multirow[t]{2}{*}{ Total } & \multicolumn{2}{|l|}{ NLR $<2.96$} & \multicolumn{2}{|l|}{$N L R \geq 2.96$} & \multirow[t]{2}{*}{$P$-value } \\
\hline & & $M L R<0.38$ & $M L R \geq 0.38$ & MLR<0.38 & MLR $\geq 0.38$ & \\
\hline Number of patients & 112 & 29 & 7 & 22 & 54 & \\
\hline Age, years & & & & & & $0.096^{\mathrm{a}}$ \\
\hline$<59$ & 50 & 14 & 4 & 5 & 27 & \\
\hline$\geq 59$ & 62 & 15 & 3 & 17 & 27 & \\
\hline Gender & & & & & & $0.872^{\mathrm{a}}$ \\
\hline Female & 61 & 14 & 4 & 13 & 30 & \\
\hline Male & 51 & 15 & 3 & 9 & 24 & \\
\hline ECOG PS & & & & & & $0.183^{a}$ \\
\hline $0-1$ & 95 & 27 & 7 & 16 & 45 & \\
\hline $2-3$ & 17 & 2 & 0 & 6 & 9 & \\
\hline Number of distant metastasis & & & & & & $0.734^{a}$ \\
\hline $0-1$ & 23 & 7 & 1 & 4 & II & \\
\hline$\geq 2$ & 89 & 22 & 6 & 18 & 43 & \\
\hline Brain metastasis & & & & & & $0.448^{a}$ \\
\hline No & 81 & 22 & 7 & 15 & 37 & \\
\hline Yes & 31 & 7 & 0 & 7 & 17 & \\
\hline Pleural effusion & & & & & & $0.37 I^{a}$ \\
\hline No & 80 & 24 & 5 & 14 & 37 & \\
\hline Yes & 32 & 5 & 2 & 8 & 17 & \\
\hline Tumor size & & & & & & $0.005^{a}$ \\
\hline$\leq 3 \mathrm{~cm}$ & 25 & 13 & 2 & 4 & 6 & \\
\hline$>3 \mathrm{~cm}$ & 87 & 16 & 5 & 18 & 48 & \\
\hline EGFR mutation type & & & & & & $0.460^{\mathrm{a}}$ \\
\hline El9del & 77 & 23 & 5 & 13 & 36 & \\
\hline L858R & 35 & 6 & 2 & 9 & 18 & \\
\hline EGFR TKI treatment & & & & & & $0.489^{a}$ \\
\hline First line & 89 & 22 & 6 & 20 & 41 & \\
\hline Second/third line & 23 & 7 & I & 2 & 13 & \\
\hline EGFR TKI type & & & & & & $0.140^{a}$ \\
\hline Erlotinib & 97 & 23 & 7 & 17 & 50 & \\
\hline Gefitinib & 15 & 6 & 0 & 5 & 4 & \\
\hline Clinical response & & & & & & $0.268^{\mathrm{a}}$ \\
\hline $\mathrm{PR}+\mathrm{SD}$ & 59 & 16 & 3 & 15 & 25 & \\
\hline PD & 53 & 13 & 4 & 7 & 29 & \\
\hline Laboratory data & & & & & & \\
\hline NEU, $10^{9} / \mathrm{L}$ & 5.99 & 4.80 & 2.53 & 7.81 & 7.55 & $<0.00 \mathrm{I}^{\mathrm{b}}$ \\
\hline$(95 \% \mathrm{Cl})$ & $(5.45-7.10)$ & $(3.75-5.45)$ & $(1.65-4.77)$ & $(6.21-9.20)$ & $(5.97-9.31)$ & \\
\hline LYM, $10^{9} / \mathrm{L}$ & 1.69 & 2.19 & 1.29 & 1.96 & 1.29 & $<0.00 I^{b}$ \\
\hline$(95 \% \mathrm{Cl})$ & $(1.45-1.95)$ & $(1.97-2.37)$ & $(0.86-2.28)$ & $(1.69-2.28)$ & $(1.08-1.53)$ & \\
\hline MONO, $10^{\circ} / \mathrm{L}$ & 0.62 & 0.57 & 0.64 & 0.57 & 0.71 & $0.025^{\mathrm{b}}$ \\
\hline$(95 \% \mathrm{Cl})$ & $(0.56-0.69)$ & $(0.45-0.68)$ & $(0.38-0.89)$ & $(0.46-0.66)$ & $(0.60-0.76)$ & \\
\hline
\end{tabular}

Notes: 'Fisher's exact test. 'Kruskal-Wallis rank test.

Abbreviations: NLR, neutrophil to lymphocyte ratio; MLR, monocyte to lymphocyte ratio; PR, partial response; SD, stable disease; PD, progressive disease; NEU, neutrophil; LYM, lymphocyte; MONO, monocyte, TKI, tyrosine kinase inhibitor; ECOG PS, Eastern Cooperative Oncology Group performance status. 
and/or chemotherapy. The EGFR TKI type used in treatment was erlotinib (Hoffman-La Roche Ltd, Basel, Switzerland) for 97 cases, with standard dose of $150 \mathrm{mg}$ /day or gefitinib (AstraZeneca plc, London, UK) for 15 cases, with standard dose of $250 \mathrm{mg} /$ day. Good response was achieved after 3 months of EGFR TKI treatment in all cases. After that, 53 cases (47.3\%) developed PD, along with various PFS, ranging from 3.9 to 28.3 months (median was 9.0 months).

\section{Median values of inflammatory index in NSCLC and control group}

The median values of inflammatory indexes in NSCLC group at baseline, after 3 months of treatment and at the PD stage are shown in Table 2 . The results demonstrated that after 3 months treatment with EGFR TKI, the WBC, NLR, MLR and PLR values of NSCLC patients were reduced considerably compared to the pretreatment values $(P<0.001)$. To answer the question of the effective of EGFR TKI in recovering the inflammatory indexes, we compared these values in NSCLC at good response stage (after 3 months of therapy) with the values of the healthy control group. The analysis has shown that the median value of WBC in NSCLC patients did not differ in statistical significance from the value of control group $(P=0.588)$. Meanwhile, the PLT, NLR, MLR and PLR values in NSCLC patients at good response stage were just slightly higher than the values of the control group $(P=0.006,0.001$, 0.003 and 0.002 , respectively) (Table 2 ).

In the other trend, we noted that the median values of inflammatory markers in NSCLC patients increased again when the disease became progressive $(P<0.001)$, and even higher than the pretreatment values (Table 2). The number of
PLT in NSCLC patients had not changed and remained statistically significant during the treatment process $(P=0.458)$.

\section{Inflammatory index in treatment monitoring for NSCLC patients}

The logistic regression model was used to construct the ROC curve of each parameter to predict the clinical response. The sensitivity, specificity and the value under the ROC curve equivalent to the optimal cutoff point of each marker were extracted and shown in Table 3.

The results shown that with the optimal cutoff point of 2.96, NLR is the best prognostic marker in predicting response to EGFR TKI among the investigated markers (AUC $=0.873, P<0.001$ ) (Figure 1 ). In addition, at this cutoff point, we can predict the clinical response with a sensitivity reached at $71.8 \%$ and a specificity reached up to $90.2 \%$ (Table 3). The results of multivariate analysis also shown that NLR is an independent predictive marker, with an OR of 3.52 (95\% CI: $1.42-8.71)(P<0.001)$.

We also recorded that MLR marker is useful in prediction of clinical response with the accuracy of $\sim 76.2 \%$ (AUC $=0.762$ ), equivalent to the optimal cutoff point of 0.38 . Despites the high specificity $(86.3 \%)$, the sensitivity of MLR in response evaluation was low (54.1\%). Treatment monitoring by the WBC, PLR or PLT markers, the AUC values of these markers were low or even very low $(0.699,0.689$ and 0.548 , respectively).

\section{Inflammatory index in prognosis for NSCLC patients}

In 112 cases, 53 cases developed progression after a median time of 9.0 (95\% CI: 7.6-10.0) months. The median PFS

Table 2 Inflammatory index values in NSCLC at different stages and in control group

\begin{tabular}{|c|c|c|c|c|c|}
\hline \multirow[t]{2}{*}{ Parameter } & \multicolumn{3}{|l|}{ NSCLC } & \multirow{2}{*}{$\begin{array}{l}\text { Control } \\
(n=I \mid 2)\end{array}$} & \multirow[t]{2}{*}{$P$-value } \\
\hline & $\begin{array}{l}\text { Baseline } \\
(n=\mid 12)\end{array}$ & $\begin{array}{l}\text { After } 3 \text { months of } \\
\text { treatment }(n=|| 2)\end{array}$ & $\begin{array}{l}\text { Progressive } \\
\text { disease } \\
(n=53)\end{array}$ & & \\
\hline $\begin{array}{l}\text { WBC, } 10^{9} / \mathrm{L} \\
(95 \% \mathrm{Cl})\end{array}$ & $\begin{array}{l}9.4 \\
(8.6-10.3)\end{array}$ & $\begin{array}{l}7.1 \\
(6.6-7.5)\end{array}$ & $\begin{array}{l}9.8 \\
(8.4-11.2)\end{array}$ & $\begin{array}{l}7.1 \\
(6.6-7.6)\end{array}$ & $\begin{array}{l}<0.001^{\mathrm{a}} \\
0.588^{\mathrm{b}}\end{array}$ \\
\hline $\begin{array}{l}\text { PLT, I0\%/L } \\
(95 \% \mathrm{Cl})\end{array}$ & $\begin{array}{l}289.9 \\
(266.3-3 \mid 3.5)\end{array}$ & $\begin{array}{l}273.4 \\
(255.7-291.2)\end{array}$ & $\begin{array}{l}271.7 \\
(248.8-294.5)\end{array}$ & $\begin{array}{l}240.7 \\
(230.6-250.8)\end{array}$ & $\begin{array}{l}0.458^{\mathrm{a}} \\
0.006^{\mathrm{b}}\end{array}$ \\
\hline $\begin{array}{l}\text { NLR } \\
(95 \% \mathrm{Cl})\end{array}$ & $\begin{array}{l}4.90 \\
(4.11-5.68)\end{array}$ & $\begin{array}{l}2.02 \\
(1.84-2.20)\end{array}$ & $\begin{array}{l}5.51 \\
(4.16-6.85)\end{array}$ & $\begin{array}{l}1.72 \\
(1.55-1.90)\end{array}$ & $\begin{array}{l}<0.001^{\mathrm{a}} \\
0.00 \mathrm{I}^{\mathrm{b}}\end{array}$ \\
\hline $\begin{array}{l}\text { MLR } \\
(95 \% \mathrm{Cl})\end{array}$ & $\begin{array}{l}0.44 \\
(0.39-0.49)\end{array}$ & $\begin{array}{l}0.28 \\
(0.26-0.31)\end{array}$ & $\begin{array}{l}0.51 \\
(0.45-0.57)\end{array}$ & $\begin{array}{l}0.20 \\
(0.18-0.22)\end{array}$ & $\begin{array}{l}<0.001^{\mathrm{a}} \\
0.003^{\mathrm{b}}\end{array}$ \\
\hline $\begin{array}{l}\text { PLR } \\
(95 \% \mathrm{Cl})\end{array}$ & $\begin{array}{l}213.9 \\
(181.9-245.9)\end{array}$ & $\begin{array}{l}143.2 \\
(131.3-155.1)\end{array}$ & $\begin{array}{l}210.3 \\
(171.6-248.9)\end{array}$ & $\begin{array}{l}108.8 \\
(101.9-115.7)\end{array}$ & $\begin{array}{l}<0.001^{\mathrm{a}} \\
0.002^{\mathrm{b}}\end{array}$ \\
\hline
\end{tabular}

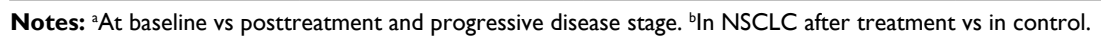

Abbreviations: NSCLC, non-small cell lung cancer; WBC, white blood cell; PLT, platelet; NLR, neutrophil to lymphocyte ratio; MLR; monocyte to lymphocyte ratio; PLR, platelet to lymphocyte ratio. 
Table 3 Optimal cutoff point of each study marker, sensitivity, specificity and AUC values in predicting response to EGFR TKI

\begin{tabular}{|c|c|c|c|c|}
\hline Parameter & Cutoff & $\begin{array}{l}\text { Sensitivity, \% } \\
(95 \% \mathrm{Cl})\end{array}$ & $\begin{array}{l}\text { Specificity,\% } \\
\text { (95\% CI) }\end{array}$ & $\begin{array}{l}\text { AUC, \% } \\
(95 \% \mathrm{CI})\end{array}$ \\
\hline WBC, $10^{9} / \mathrm{L}$ & 9.0 & $\begin{array}{l}48.2 \\
(37.3-59.3)\end{array}$ & $\begin{array}{l}78.4 \\
(69.2-86.0)\end{array}$ & $\begin{array}{l}0.699 \\
(0.623-0.775)\end{array}$ \\
\hline PLT, I0\%/L & 324.0 & $\begin{array}{l}32.9 \\
(23.1-44.0)\end{array}$ & $\begin{array}{l}77.5 \\
(68.1-85.1)\end{array}$ & $\begin{array}{l}0.548 \\
(0.464-0.632)\end{array}$ \\
\hline NLR & 2.96 & $\begin{array}{l}71.8 \\
(60.5-81.4)\end{array}$ & $\begin{array}{l}90.2 \\
(82.6-95.5)\end{array}$ & $\begin{array}{l}0.873 \\
(0.821-0.926)\end{array}$ \\
\hline MLR & 0.38 & $\begin{array}{l}54.1 \\
(44.7-67.6)\end{array}$ & $\begin{array}{l}86.3 \\
(77.5-92.4)\end{array}$ & $\begin{array}{l}0.762 \\
(0.691-0.832)\end{array}$ \\
\hline PLR & 189.8 & $\begin{array}{l}49.4 \\
(38.4-60.5)\end{array}$ & $\begin{array}{l}78.4 \\
(69.2-86.0)\end{array}$ & $\begin{array}{l}0.689 \\
(0.613-0.766)\end{array}$ \\
\hline
\end{tabular}

Abbreviations: AUC, area under the curve; WBC, white blood cell; PLT, platelet; NLR, neutrophil to lymphocyte ratio, MLR, monocyte to lymphocyte ratio; PLR, platelet to lymphocyte ratio; TKI, tyrosine kinase inhibitor.

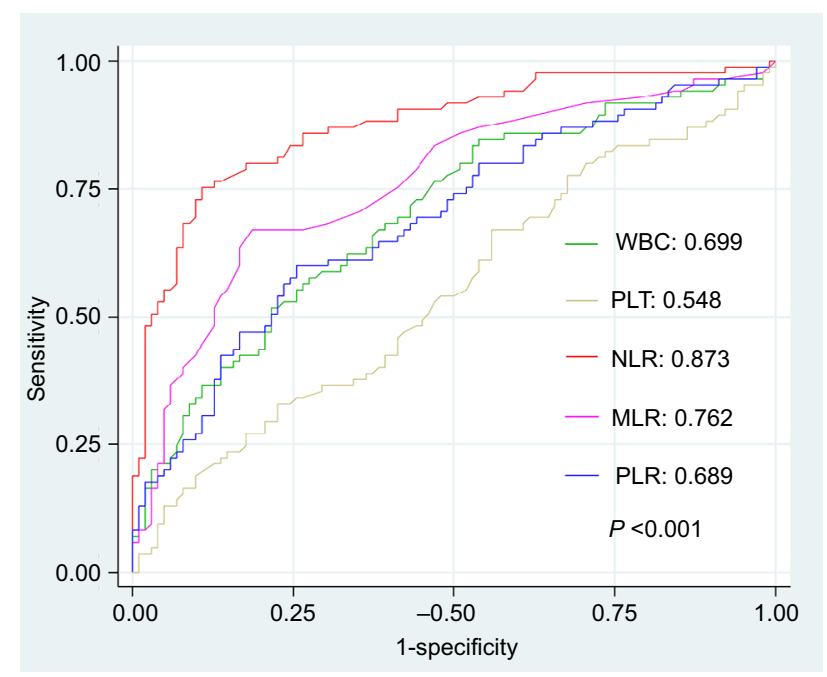

Figure I Comparison of area under the ROC curve of inflammatory markers in response evaluation.

Abbreviations: WBC, white blood cell; PLT, platelet; NLR, neutrophil to lymphocyte ratio, MLR, monocyte to lymphocyte ratio; PLR, platelet to lymphocyte ratio; ROC, receiver operating characteristic.

and HR based on subgroups of pretreatment inflammatory markers and clinical characteristics were estimated by the Kaplan-Meier and Cox regression methods (Table 4).

The results of univariate analysis shown that the tumor size and NLR were two parameters that help to predict the clinical outcome for NSCLC patients treated with EGFR TKI (Figure 2). Of 53 resistant patients, 44 patients $(83.0 \%)$ have the tumor size $>3 \mathrm{~cm}$. The median PFS of patients with tumor size $>3$ $\mathrm{cm}$ was 8.2 (95\% CI: 7.5-10.4) months, whereas the value of patients with tumor size $\leq 3 \mathrm{~cm}$ was 13.6 (95\% CI: 5.8-23.3) months. This difference was statistically significant $(\mathrm{HR}=2.24$, 95\% CI: 1.04-4.83) ( $P=0.039)$. The median PFS of patients with pretreatment NLR $\geq 2.96(\mathrm{n}=36 / 53)$ was only around 7.7 (95\% CI: 6.0-8.1) months, which was shorter than the value of the $\mathrm{NLR}<2.96$ groups ( $\mathrm{PFS}=11.1,95 \% \mathrm{CI}$ : $9.5-13.9$ months), HR=2.67 (95\% CI: 1.41-5.03) ( $P=0.006)$. Whereas the median PFS of patients with high level of pretreatment MLR ( $\geq 0.38)(n=32 / 53)$ was 7.5 (95\% CI: 5.9-8.4) months, the median PFS in patients with low level of MRL was 10.1 (95\% CI: 9.0-13.0) months, HR=1.91 (95\% CI: 1.07-3.41) $(P=0.034)$. However, the crossover between survival curves at the end of the Kaplan-Meier plot (Figure 2C) indicates that the difference of PFS between groups of the lower and higher MLR level might not be significant. We checked this with the proportional hazards assumption tests and confirmed that MLR is not a significant prognostic factor in this situation (the log-log plot shown the crossed lines while the observed values and predicted values are different; figures not shown). No differences of PFS between groups of age, gender, ECOG PS, number of distant metastasis, status of brain metastasis, pleural effusion, EGFR mutation type, EGFR TKI type, and the level of WBC, PLT and PLR were recorded.

In multivariate analysis, we noted that the elevated NLR is an independent prognostic marker for worse PFS of EGFRmutant NSCLC patients treated with EGFR TKI, HR=2.15 (95\% CI: 1.15-3.99) $(P=0.016)$ (Table 4).

\section{Discussion}

In the past 10 years (2009-2018), more than 20 studies have focused on evaluating the roles of inflammatory markers in NSCLC. ${ }^{12}$ Most of these studies have been performed on the patients treated with surgery, chemotherapy or radiotherapy. Relatively few studies have been conducted on patients treated with targeted therapies, especially with EGFR TKI. ${ }^{13-18}$ In these studies, the authors also show the different viewpoints about the role of inflammatory markers, especially NLR and lymphocyte to monocyte ratio in prognosis for NSCLC patients treated with EGFR TKI. ${ }^{13-18}$ 
Table 4 Estimation of PFS and prognosis markers for NSCLC patients

\begin{tabular}{|c|c|c|c|c|c|}
\hline \multirow[t]{2}{*}{ Parameters } & \multirow[t]{2}{*}{ PFS (95\% Cl) } & \multicolumn{2}{|c|}{ Univariate analysis } & \multicolumn{2}{|c|}{ Multivariate analysis } \\
\hline & & HR (95\% CI) & $P$-value & HR $(95 \% \mathrm{Cl})$ & $P$-value \\
\hline Age, years & & $0.96(0.55-1.68)$ & 0.897 & - & - \\
\hline$<59$ & $9.0(7.5-11.3)$ & & & & \\
\hline$\geq 59$ & $8.8(7.7-13.0)$ & & & & \\
\hline Gender & & $1.13(0.65-1.98)$ & 0.658 & - & - \\
\hline Female & $8.8(7.5-12.0)$ & & & & \\
\hline Male & $9.0(7.9-11.3)$ & & & & \\
\hline ECOG PS & & $1.91(0.84-4.33)$ & 0.121 & - & - \\
\hline $0-1$ & $9.0(8.0-11.3)$ & & & & \\
\hline $2-3$ & $7.9(3.2-11.1)$ & & & & \\
\hline Number of distant metastasis & & $2.01(0.79-5.10)$ & 0.142 & - & - \\
\hline $0-1$ & $10.4(5.8-12.1)$ & & & & \\
\hline$\geq 2$ & $8.8(7.9-11.0)$ & & & & \\
\hline Brain metastasis & & $1.56(0.84-2.91)$ & 0.160 & - & - \\
\hline No & $9.2(7.9-12.0)$ & & & & \\
\hline Yes & $8.0(6.2-11.0)$ & & & & \\
\hline Pleural effusion & & $1.55(0.8 \mathrm{I}-2.98)$ & 0.191 & - & - \\
\hline No & $9.2(8.0-11.3)$ & & & & \\
\hline Yes & $7.7(3.1-12.2)$ & & & & \\
\hline Tumor size & & $2.24(1.04-4.83)$ & 0.039 & - & - \\
\hline$\leq 3 \mathrm{~cm}$ & $13.6(5.8-23.3)$ & & & & \\
\hline$>3 \mathrm{~cm}$ & $8.2(7.5-10.4)$ & & & & \\
\hline EGFR mutation type & & $0.84(0.49-1.46)$ & 0.546 & - & - \\
\hline El9del & $9.0(7.7-12.2)$ & & & & \\
\hline L858R & $8.0(6.2-11.1)$ & & & & \\
\hline EGFR TKI treatment & & $0.96(0.61-1.79)$ & 0.802 & - & - \\
\hline First line & $8.8(7.5-11.0)$ & & & & \\
\hline Second/third line & $10.4(7.5-13.8)$ & & & & \\
\hline EGFR TKI type & & $0.43(0.28-1.02)$ & 0.506 & - & - \\
\hline Erlotinib & $8.2(7.7-10.4)$ & & & & \\
\hline Gefitinib & $11.0(5.5-23.3)$ & & & & \\
\hline WBC, $10^{\circ} / \mathrm{L}$ & & $1.65(0.92-2.96)$ & 0.325 & - & - \\
\hline$<9.0$ & $9.3(7.9-11.2)$ & & & & \\
\hline$\geq 9.0$ & $8.0(7.1-9.9)$ & & & & \\
\hline PLT, $10^{9} / \mathrm{L}$ & & $0.92(0.50-1.72)$ & 0.612 & - & - \\
\hline$<324.0$ & $9.0(7.9-10.0)$ & & & & \\
\hline$\geq 324.0$ & $8.0(6.9-14.0)$ & & & & \\
\hline NLR & & $2.67(I .4 \mid-5.03)$ & 0.006 & $2.15(1.15-3.99)$ & 0.016 \\
\hline$<2.96$ & 11.1 (9.5-13.9) & & & & \\
\hline$\geq 2.96$ & $7.7(6.0-8.1)$ & & & & \\
\hline MLR & & 1.91 (I.07-3.4I) & 0.034 & - & - \\
\hline$<0.38$ & $10.1(9.0-13.0)$ & & & & \\
\hline$\geq 0.38$ & $7.5(5.9-8.4)$ & & & & \\
\hline PLR & & $1.09(0.62-1.92)$ & 0.764 & - & - \\
\hline$<189.8$ & $9.1(8.0-10.4)$ & & & & \\
\hline$\geq 189.8$ & $7.9(5.4-10.7)$ & & & & \\
\hline
\end{tabular}

Abbreviations: PFS, progression-free survival; NSCLC, non-small cell lung cancer; WBC, white blood cell; PLT, platelet; NLR, neutrophil to lymphocyte ratio; MLR, monocyte to lymphocyte ratio; PLR, platelet to lymphocyte ratio; TKI, tyrosine kinase inhibitor; ECOG PS, Eastern Cooperative Oncology Group performance status.

Through the study on 112 NSCLC patients treated with EGFR TKI, we took note that the WBC, NLR, MLR and PLR values have been reduced significantly in patients with good response after 3 months compared with pretreatment. The results also show that at the good response stage, these markers in NSCLC patients are nearly equivalent to the same markers of healthy adults. Under the pressure of medicine for targeted therapy, such as erlotinib or gefitinib, hypersensitive tumor cells were annihilated, parallel with reducing the secretion of some cytokines such as granulocyte-macrophage colony 
A

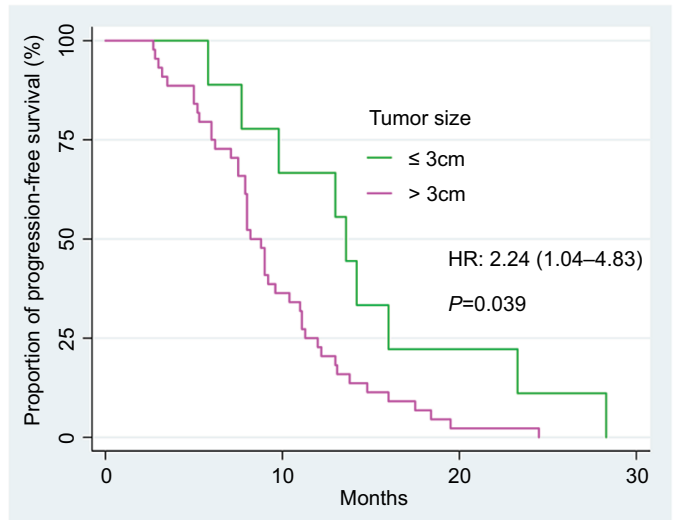

B

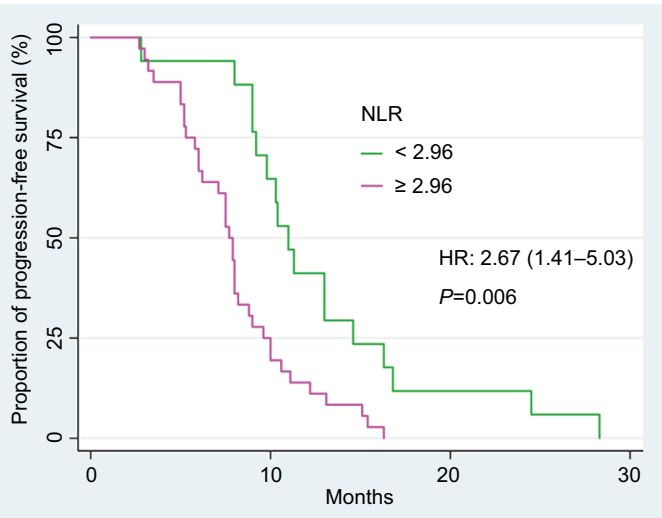

C

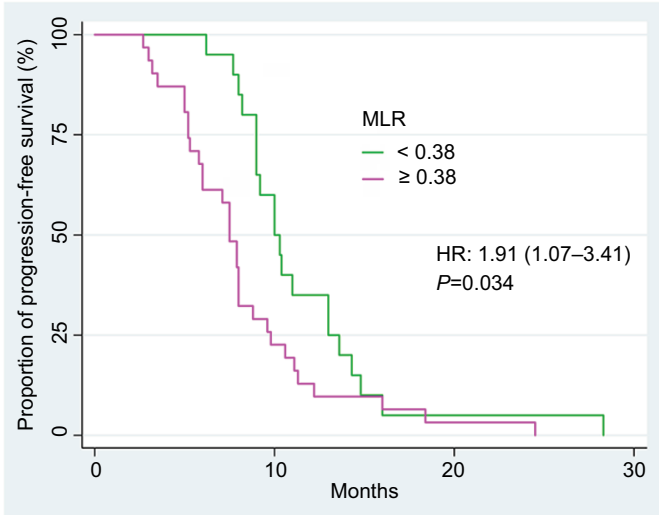

Figure 2 Progression-free survival of NSCLC patients according to the tumor size (A), the pretreatment levels of NLR (B), and MLR (C).

Abbreviations: NLR, neutrophil to lymphocyte ratio; MLR, monocyte to lymphocyte ratio; NSCLC, non-small cell lung cancer.

stimulating factor, granulocyte colony stimulating factor, IL-1 or IL-6, thus the inflammatory markers tend to return to normal. ${ }^{20}$ We also found out the decrease of NLR and MLR helped to predict the ability of good response to EGFR TKI, in which NLR is an independent predictive marker. Moreover, together with an accuracy of over $87 \%$, sensitivity reached at good level (71\%) and high specificity (90\%), we believe that these markers are very useful in response evaluation for NSCLC patients. Compared with other serum markers as cyfra 21-1, CA12-5, NSE or imaging diagnostic tools as PET-CT, CT-scanner or MRI, the use of inflammatory markers as NLR and MLR in treatment monitoring will ensure the safety of patients while maintaining low cost and being easy to implement. However, we assume that the use of these inflammatory indexes in combination with conventional serum biomarkers might help to increase the accuracy in prediction of clinical response. We have not selected enough data of conventional biomarkers for further analysis. Large-scale research should be conducted to clarify this hypothesis.

In this study, we also found that the inflammatory markers tended to increase conversely when the disease develops progression, and even more higher compared to pretreatment values. The tumor cells that are resistant to EGFR TKI survived posttreatment; they will keep growing and return to develop strongly. The tumor will increase production of the differentiation markers of WBCs, attract, control and utilize neutrophil or monocyte in a manner benefitting the development. ${ }^{20}$ Based on this principle, the high level of inflammatory index is an indicator in predicting the risk of early resistance. Some studies show that having various prognostic models for NSCLC, patients such as the combination of NEU with MONO, NLR with prognostic nutritional index, NLR with PLR, and NLR with PL. ${ }^{10}$ We noted that MLR, PLT and PLR did not have significance in prognosis for NSCLC patients. Meanwhile, the larger tumor size $(>3 \mathrm{~cm})$ and the high level of pretreatment NLR have been closely associated with the shortening of PFS. In addition, we confirmed that NLR is an independent prognostic marker for NSCLC patients treated with EGFR TKI which is similar to the results of three previous studies. ${ }^{13-15}$ However, this is contrary to the results of Minami et al, and Sim et al. ${ }^{16,18}$ There are some differences between our study and these two studies. The patient age of our study was lower (59 vs 70 and 67 years, respectively), whereas the proportion of male patients was higher $(45.5 \%$ vs $37.5 \%$ and $30.6 \%$, respectively) than in 
these two studies. The lower proportion of severely ill patients (ECOG PS $\geq 2$ ) was also observed in our study (15.2\% vs $27 \%$ and $30.6 \%$, respectively). These factors might affect the pretreatment NLR value, and thus affect the prognostic models that lead to the different results. Besides, the proportion of EGFR TKI as first-line treatment, the cutoff value, or even the blood cell count method might be the bias factors in prognostic models that should be considered in a large-scale study.

In conclusion, the results of this study indicated that NLR and MLR are valuable inflammatory markers in response evaluation for NSCLC patients treated with EGFR TKI. Moreover, the elevated NLR is an independent prognostic marker for poor survival.

\section{Author contributions}

Son Truong Nguyen is the senior author who contributed to study design; Thang Thanh Phan and Hang Thuy Nguyen collected the clinical data; Toan Trong Ho and Hue Thi Nguyen collected the laboratory data; Thang Thanh Phan and Thu Bich Tran performed the data analysis and wrote the manuscript. All authors contributed toward data analysis, drafting and critically revising the paper, gave final approval of the version to be published, and agree to be accountable for all aspects of the work.

\section{Disclosure}

The authors report no conflicts of interest in this work.

\section{References}

1. Fitzmaurice C, Allen C, Barber RM, et al. A systematic analysis for the global burden of disease study. JAMA Oncol. 2017;3(4):524-548.

2. Kumarakulasinghe NB, van Zanwijk N, Soo RA. Molecular targeted therapy in the treatment of advanced stage non-small cell lung cancer (NSCLC). Respirology. 2015;20(3):370-378.

3. Ahn JM, Cho JY. Current serum lung cancer biomarkers. J Mol Biomark Diagn. 2013;S4(001).

4. Kemal Y, Yucel I, Ekiz K, et al. Elevated serum neutrophil to lymphocyte and platelet to lymphocyte ratios could be useful in lung cancer diagnosis. Asian Pac J Cancer Prev. 2014;15(6):2651-2654.

5. Botta C, Barbieri V, Ciliberto D, et al. Systemic inflammatory status at baseline predicts bevacizumab benefit in advanced non-small cell lung cancer patients. Cancer Biol Ther. 2013;14(6):469-475.

6. Kos FT, Hocazade C, Kos M, et al. Assessment of prognostic value of "neutrophil to lymphocyte ratio" and "prognostic nutritional index" as a sytemic inflammatory marker in non-small cell lung cancer. Asian Pac J Cancer Prev. 2015;16(9):3997-4002.

International Journal of General Medicine

Publish your work in this journal

The International Journal of General Medicine is an international, peer-reviewed open-access journal that focuses on general and internal medicine, pathogenesis, epidemiology, diagnosis, monitoring and treatment protocols. The journal is characterized by the rapid reporting of reviews, original research and clinical studies across all disease areas.
7. Wu G, Yao Y, Bai C, et al. Combination of platelet to lymphocyte ratio and neutrophil to lymphocyte ratio is a useful prognostic factor in advanced non-small cell lung cancer patients. Thorac Cancer. 2015;6(3):275-287.

8. Yao Y, Yuan D, Liu H, Gu X, Song Y. Pretreatment neutrophil to lymphocyte ratio is associated with response to therapy and prognosis of advanced non-small cell lung cancer patients treated with firstline platinum-based chemotherapy. Cancer Immunol Immunother. 2013;62(3):471-479.

9. Lee Y, Kim SH, Han JY, Kim HT, Yun T, Lee JS. Early neutrophil-tolymphocyte ratio reduction as a surrogate marker of prognosis in never smokers with advanced lung adenocarcinoma receiving gefitinib or standard chemotherapy as first-line therapy. J Cancer Res Clin Oncol. 2012;138(12):2009-2016.

10. Zhang $\mathrm{H}$, Zhang $\mathrm{L}$, Zhu K, et al. Prognostic significance of combination of preoperative platelet count and neutrophil-lymphocyte ratio (COP-NLR) in patients with non-small cell lung cancer: based on a large cohort study. PLoS One. 2015;10(5):e0126496.

11. Lin GN, Peng JW, Xiao JJ, Liu DY, Xia ZJ. Prognostic impact of circulating monocytes and lymphocyte-to-monocyte ratio on previously untreated metastatic non-small cell lung cancer patients receiving platinum-based doublet. Med Oncol. 2014;31(7):70.

12. Zhao QT, Yang Y, Xu S, et al. Prognostic role of neutrophil to lymphocyte ratio in lung cancers: a meta-analysis including 7,054 patients. Onco Targets Ther. 2015;8:2731-2738.

13. Lin GN, Peng JW, Liu PP, Liu DY, Xiao JJ, Chen XQ. Elevated neutrophil to lymphocyte ratio predicts poor outcome in patients with advanced non-small cell lung cancer receiving first-line gefitinib or erlotinib treatment. Asia Pac J Clin Oncol. 2014.

14. Ding PN, Roberts TL, Chua W, et al. Clinical outcomes in patients with advanced epidermal growth factor receptor-mutated non-small-cell lung cancer in South Western Sydney Local Health District. Intern Med J. 2017;47(12):1405-1411.

15. Meriggi F, Codignola C, Beretta GD, et al. Significance of neutrophil-to-lymphocyte ratio in Western advanced EGFR-mutated non-small cell lung cancer receiving a targeted therapy. Tumori. 2017;103(5):443-448.

16. Minami S, Ogata Y, Ihara S, Yamamoto S, Komuta K. Neutrophil-tolymphocyte ratio predicts overall survival of advanced non-small cell lung cancer harboring mutant epidermal growth factor receptor. World J Oncol. 2017;8(6):180-187.

17. Chen Y-M, Lai C-H, Chang H-C, et al. Baseline and trend of lymphocyteto-monocyte ratio as prognostic factors in epidermal growth factor receptor mutant non-small cell lung cancer patients treated with firstline epidermal growth factor receptor tyrosine kinase inhibitors. PLoS One; $10(8): \mathrm{e} 0136252$.

18. Sim SH, Beom SH, Ahn YO, et al. Pretreatment neutrophil-lymphocyte ratio is not a significant prognostic factor in epidermal growth factor receptor-mutant non-small cell lung cancer patients treated with tyrosine kinase inhibitors. Thorac Cancer. 2016;7(2):161-166.

19. Eisenhauer EA, Therasse P, Bogaerts J, et al. New response evaluation criteria in solid tumours: revised RECIST guideline (version 1.1). Eur J Cancer. 2009;45(2):228-247.

20. Uribe-Querol E, Rosales C. Neutrophils in cancer: two sides of the same coin. J Immunol Res. 2015;2015:983698-21.
The manuscript management system is completely online and includes a very quick and fair peer-review system, which is all easy to use. Visit http://www.dovepress.com/testimonials.php to read real quotes from published authors. 